Recibido: 01/08/2020 --- Aceptado: 19/02/2021 --- Publicado: 12/04/2021

\title{
EI USO DE REDES SOCIALES EN MICROEMPRESAS ANTE EFECTOS COVID-19
}

\section{THE USE OF SOCIAL MEDIA IN MICRO-ENTERPRISES IN THE FACE OF COVID-19 EFFECTS}

(iD) 8 María del Rosario Demuner Flores ${ }^{1}$. Universidad Autónoma del Estado de México. México.

mrdemunerf@uaemex.mx

Agradecimientos. Esta investigación fue realizada con el apoyo del Sr. Arcadio Andrade, Administrador del grupo WhatsApp "El Mercado Virtual de las y los Cordobeses", \#AquisiNosapoyamos. Y el presidente de CANACO SERVYTUR CORDOBA, Ing. Carlos Lara Álvarez.

\section{Cómo citar el artículo:}

Demuner Flores, M. R. (2021). Uso de redes sociales en microempresas ante efectos COVID-19. Revista de Comunicación de la SEECI, 54, 97-118. https://doi.org/10.15198/seeci.2021.54.e660

\section{RESUMEN}

La economía mundial sufre una crisis sanitaria y económica donde la vulnerabilidad de la MIPYME es evidente. El objetivo fue analizar el uso de las redes sociales que eligieron las microempresas en respuesta a los efectos causados por la pandemia COVID-19 en una localidad mexicana. El estudio transversal con técnica narrativa describe los resultados de una encuesta a empresarios. El confinamiento social y el cierre obligatorio afectaron la actividad de las microempresas (48\%), provocaron disminución de sus ingresos (31\%), incrementaron las necesidades de financiamiento (24\%) y obligaron al despido de empleados (16\%). En respuesta a los efectos causados, la estrategia fue incrementar $30 \%$ el uso de redes sociales. Principalmente Facebook y WhatsApp se usaron para promoción, seguimiento de clientes, posicionar productos y explorar mercados. La principal aportación de esta investigación es evidenciar empíricamente los efectos de la pandemia y el uso de redes sociales como alternativa de las microempresas para lograr su subsistencia.

\footnotetext{
${ }^{1}$ María del Rosario Demuner Flores: Profesora investigadora de la Facultad de Contaduría y Administración de la Universidad Autónoma del Estado de México, miembro del Sistema Nacional de Investigadores, líneas de investigación: Gestión del capital intelectual, competitividad PYME. Directora de RECAI Revista de Estudios en Contaduría, Administración e Informática. mrdemunerf@uaemex.mx
} 
PALABRAS CLAVE: -Redes sociales - Digitalización- Crisis económica Microempresas - COVID-19

\section{ABSTRACT}

The world economy suffers a health and economic crisis where the vulnerability of SMEs is evident. The purpose was to analyze the use of social networks chosen by micro-enterprises in response to the effects caused by the COVID-19 pandemic in a Mexican town. The cross-sectional study with narrative technique describes the results of a survey of entrepreneurs. Social confinement and mandatory closure affected the activity of the micro-enterprises (48\%), caused a decrease in its income $(31 \%)$, increased financing needs $(24 \%)$ and forced the dismissal of employees (16\%). In response to the effects caused, the strategy was to increase the use of social networks $30 \%$. Mainly Facebook and WhatsApp were used for promotion, customer tracking, positioning products and exploring markets. The main contribution is to empirically evidence the effects of the pandemic and the use of social networks as an alternative for micro-enterprises to achieve their survival.

KEY WORDS: - Social networks - Digitization- Economic crisis - Micro-enterprises. COVID-19

\section{O USO DAS REDES SOCIAIS EM MICROEMPRESAS PERANTE EFEITOS DO COVID-19}

\section{RESUMO}

A economia mundial sofre uma crise sanitária e econômica onde a vulnerabilidade das PIME é evidente. $\mathrm{O}$ objetivo foi analisar o uso das redes sociais que escolheram as microempresas como resposta aos efeitos causados pela pandemia COVID-19 em uma localidade mexicana. $O$ estudo foi transversal com a técnica narrativa que descreve os resultados de um questionário a empresários. O confinamento social e o fechamento obrigatório afetaram a atividade das microempresas (48\%), provocaram a diminuição dos seus ingressos (31\%), incrementaram as necessidades de financiamento (24\%) e obrigaram a demissão de empregados (16\%). Em resposta aos efeitos causados, a estratégia foi incrementar $30 \%$ o uso das redes sociais. Principalmente Facebook e WhatsApp se usaram para promoção, segmento de clientes, posicionar produtos e procurar novos mercados. O principal aporte desta pesquisa é evidenciar empiricamente os efeitos da pandemia e o uso das redes sociais como alternativa das microempresas para atingir sua subsistência.

PALABRAS CLAVE: - Redes sociais - Digitalização - Crise econômica Microempresas - COVID-19 


\section{INTRODUCCIÓN}

La economía en el mundo sufre una crisis sanitaria derivada de los efectos de la pandemia COVID-19, a la fecha ha cobrado numerosas vidas. La posibilidad de contagio ha llevado a los gobiernos a implementar medidas drásticas, como el aislamiento social, fuerte inversión en equipamiento médico y el cierre de empresas que han paralizado la economía mundial.

El Observatorio COVID-19 en América Latina y el Caribe, de la CEPAL, informa sobre la situación y las medidas de política que ha establecido para enfrentar la crisis. La acción más importante que ha sugerido para aplanar la curva de contagio y enfrentar la pandemia, es la contención de la expansión del virus mediante el autoaislamiento, la cuarentena y el distanciamiento social. Medidas sanitarias, que afectan la economía, en cuestión a horas de trabajo, salarios, cierre de fábricas, despidos de personal, disminución en inversiones privadas, cese de operaciones de servicios públicos, cancelación de actividades y eventos sociales, entre otros (CEPAL, 2020).

La magnitud del choque de demanda agregada debido a la reducción del consumo de bienes y servicios provocado por la pandemia se ha convertido en la mayor crisis económica que el mundo ha experimentado desde los años 30's (FMI, 2020). Como resultado de la incertidumbre, la demanda interna ha provocado la disminución de liquidez, la paralización de la actividad económica, disrupciones en las cadenas de pago, pérdidas de rentabilidad y riqueza, volatilidad de los mercados financieros, incluso la paralización económica en algunas regiones (CEPAL, 2020).

América Latina y el Caribe han enfrentado la pandemia desde una posición más débil que la del resto del mundo. La región ha sufrido una disminución de la actividad económica con sus principales socios comerciales; los efectos se traducen en la caída de los precios de sus productos primarios, interrupción de cadenas globales de valor como, los sectores manufactureros que en México y Brasil son los más grandes de la región, el turismo, y las condiciones financieras. Las medidas de distanciamiento social han afectado a los sectores de distintas formas, algunos han sido más dañados que otros. Por ejemplo, los servicios que, en gran medida, dependen de contactos interpersonales, como el transporte, turismo, entretenimiento, empleo informal y la manufactura porque difícilmente se realiza a distancia (CEPAL, 2020).

Durante 2020, debido a COVID-19, México sufrió una caída del PIB de 8.3\% (INEGI, 2020). Las consecuencias se desbordaron en la disminución del envió de remesas, en la caída de los precios de las materias primas, disminución del ingreso del turismo, incremento en la aversión al riesgo por parte de los inversionistas internacionales, aumento de las salidas de capitales, volatilidad de los mercados financieros y detrimento en la actividad industrial (-3.3\%) (INEGI, 2020a). En marzo 2020, la industria automotriz mexicana anunció una caída del 30\% comparado con marzo 2019 (AMIA, 2020), resultado que provocó el cierre de su cadena de proveeduría -una de las más importantes en México por su cercanía con uno de sus

Revista de Comunicación de la SEECI. 2021, nº 54, 97-118 
mejores clientes, Estados Unidos- debido a la carencia de insumos esenciales y al confinamiento de los trabajadores a sus hogares (INEGI, 2020). Resultados también atribuidos a la crisis sanitaria provocada por COVID-19.

La crisis afectó al sector empresarial mexicano en sus ingresos, solvencia y pérdida de puestos de trabajo. La tasa de desempleo subió a 5,5\% en junio 2020, el total de empleos en riesgo alto rebasó los 24 millones (44\% del empleo total en México) (OIT, 2020). Algunos grupos poblacionales fueron más vulnerables, entre ellos se ubica a las micro, pequeñas y medianas empresas (MIPYME). Las MIPYME resintieron la caída de sus ventas, en parte, por efectos del aislamiento social y también por las disposiciones gubernamentales de cierre obligatorio. Sin embargo, algunas de ellas, replantearon su actividad, acudieron al uso masivo de redes sociales como alternativa de intercambio comercial y para promoción de sus productos ante la contingencia vivida (González y Flores, 2020). Mientras que las grandes empresas pueden permitirse quedarse quietas y ser resilientes, las MIPYME deben adaptarse de forma ágil si no desean derrumbarse. A causa de COVID-19, el $34 \%$ de las empresas mexicanas con menos de 10 empleados y el $26 \%$ de quienes emplean entre 10 y 20 personas, tuvieron un impacto catalogado como "grave" (Konfio, 2020).

Por su parte, los microempresarios de Córdoba Veracruz también recibieron la afectaciones provocadas por la contingencia sanitaria. Sus decisiones de cerrar sus establecimientos y despedir empleados, no se hizo esperar. Sin embargo, algunos empresarios adecuaron sus actividades de atención promoción comercialización y distribución. Se unieron en grupos y emplearon redes sociales para difundir sus productos y servicios. Además de obtener ingresos -aunque no en la cantidad que esperaban-, lograron beneficiar una sociedad confinada a permanecer en sus hogares, donde el abastecimiento de productos de primera necesidad se convirtió en un inconveniente. La intensión del uso de redes sociales (RS) se adhiere a la inclusión de esas tecnologías relacionadas con todo tipo de modalidades de comunicación que derivan del Internet, y que para la literatura científica significan un gran beneficio para fortalecer los resultados de las empresas (Rodríguez y Da Cunha, 2018).

En esta línea, el objetivo de la presente investigación es analizar el uso de las redes sociales que eligieron las microempresas en respuesta a los efectos causados por la pandemia COVID-19 en una localidad mexicana. Se presenta después de la introducción, el escenario en que se desenvuelven las micro, pequeñas y medianas empresas mexicanas; conceptualización de las redes sociales y sus implicaciones; metodología empleada en este estudio; resultados encontrados y su respectiva discusión; para terminar, mencionando algunas conclusiones.

\section{REVISIÓN CONTEXTUAL Y TEÓRICA}

\subsection{Contexto MIPYME}


Las MIPYME juegan un papel muy importante dentro de la economía mexicana, durante 2019 representaron el $99.8 \%$ de las empresas y participaron con el $67.9 \%$ en el empleo (INEGI, 2019). En el Estado de Veracruz en 2019, las MIPYME ocuparon un universo del $99.9 \%$ y también fueron la principal fuente de empleo (85.2\%) (Tabla 1).

Tabla 1. Unidades económicas y personal ocupado a nivel nacional y Estado de Veracruz.

\begin{tabular}{crrrr}
\hline Unidades & $\begin{array}{r}\text { Personal } \\
\text { ocupado }\end{array}$ \\
\hline económicas & $4,800,157$ & $100 \%$ & $27,132,927$ & $100 \%$ \\
Motal nacional & $4,555,263$ & $94.9 \%$ & $10,086,104$ & $37.2 \%$ \\
Pequeña & 193,423 & $4.0 \%$ & $4,022,649$ & $14.8 \%$ \\
Mediana & 40,824 & $0.9 \%$ & $4,309,526$ & $15.9 \%$ \\
Grande & 10,647 & $0.2 \%$ & $8,714,648$ & $32.1 \%$ \\
& & & & \\
Total Veracruz & 438,213 & $100 \%$ & $1,701,915$ & $100 \%$ \\
Micro & 418,766 & $95.6 \%$ & 867,116 & $50.9 \%$ \\
Pequeña & 16,547 & $3.8 \%$ & 331,309 & $19.5 \%$ \\
Mediana & 2,511 & $0.6 \%$ & 252,192 & $14.8 \%$ \\
Grande & 389 & $0.1 \%$ & 251,298 & $14.8 \%$ \\
\hline
\end{tabular}

Nota: Datos 2018.

Fuente: Elaboración propia en base a INEGI (2019)

El municipio de Córdoba se encuentra en la cuarta posición en el Estado de Veracruz. Participa con el $4.7 \%$ de las empresas en el Estado, su contribución al empleo fue $5.1 \%$ con remuneraciones de $3.4 \%$, su producción bruta total fue $3.5 \%$, el valor agregado censal bruto $3.1 \%$ y en el total de activos fijos aportó el $1.6 \%$ (INEGI, 2014).

En Córdoba, Estado de Veracruz, las MIPYME conforman alrededor del $98 \%$ de las empresas -las de tamaño micro tienen la mayor representación-, en promedio albergan a 4 personas, la remuneración media anual por persona en miles de pesos es de 69.9, la producción bruta total por persona ocupada en miles de pesos es de 502.8, los activos fijos por persona ocupada corresponde en miles de pesos a 140.2 y la maquinaria y equipo por persona ocupada en miles de pesos es de 59.6, siendo el margen operativo del municipio 18.2\% (INEGI, 2014).

Al igual que a nivel nacional, en Córdoba, este grupo de empresas son la principal fuente de empleo y autoempleo, además de albergar la mano de obra no especializada a pesar de algunas limitaciones en su desempeño, como la accesibilidad al financiamiento, la falta de planeación, alta tasa de natalidad y mortalidad, contabilidad no profesionalizada y resistencia al cambio (Demuner, 2011). La situación económica de las MIPYME en Córdoba también se agravó con la presencia de la pandemia. En general, en México el cierre temporal de algunas 
empresas y el cierre definitivo de otras afectó el tejido social dejando en el desamparo a muchas familias. Especialmente, este grupo de empresas agotó sus reservas de efectivo. En respuesta, el gobierno instrumentó medidas de ayuda, como financiamiento a través de créditos con bajas tasas de interés, adelantar capital para nuevos proyectos, agilizar las devoluciones de IVA, posponer o diferir el pago de algunos impuestos $\mathrm{y} / \mathrm{o}$ suspender temporalmente el pago de algunos servicios (ALAMPYME, 2020).

El estímulo no fue solo la implementación de políticas gubernamentales en ayuda a la MIPYME, los propietarios de las empresas sobrevivientes respondieron con estrategias dirigidas a un trabajo más eficiente, a un cambio en la forma de hacer las cosas, como, por ejemplo, incursionar en el comercio electrónico. Asumir conciencia sobre el camino ayudó a responder a la situación, replantearon actividades, redujeron y optimizaron costos y gastos, pensaron en nuevos modelos de negocio, incursionaron en otros nichos de mercado, intentaron cambiar su producto o servicio por otro necesario en ese momento. Estas accionen corresponden propiamente al desarrollo de su know how. El cúmulo de conocimiento y experiencia permite a la pequeña empresa mejorar sus actividades de gestión y contribuir de forma significativa a lograr la competitividad (Ibarra, González y Demuner, 2017).

Responder a las exigencias de un mercado globalizado insta a las MIPYME a repensar sus estrategias para enfrentar el riesgo en función de la competitividad, el mercado y la sostenibilidad (Pereira y Cuero, 2018). La implementación y revisión periódica de sus acciones, y la coherencia en el actuar con los objetivos planteados para enfocarse a la turbulencia del entorno, implica desarrollar un conjunto de habilidades y rutinas diferenciadas que las guíe para reaccionar de forma rápida y más eficiente ante los cambios del ambiente (Demuner, Becerril e Ibarra, 2018).

El perfil del microempresario mexicano incluye una estructura de valores provenientes del entorno en que se desenvuelve, que explican el dinamismo y la permanencia de sus empresas. A veces, aprovechan los espacios naturales para desarrollar el papel de emprendedores (Ugalde, 2009). Otras veces, estos empresarios se manifiestan mediante la necesidad de auto emplearse y crear su propio negocio donde cobijan a familiares y amigos; existe evidencia de que ambos grupos, constituyen el personal más importante para su operatividad (Zúñiga, Soza y Soria, 2015).

Es común percibir empresas de tamaño micro que surgen de la iniciativa de alguien que domina alguna habilidad técnica para fabricar algún producto, pero desconoce tácticas administrativas. Ibarra et al. (2017) realizaron un estudio sobre la competitividad de las MIPYME de Baja California en México, y encontraron que las empresas que elaboran sus productos dirigen más su atención a la producción y operaciones relacionadas con el proceso de manufactura; y descuidan actividades de planeación, contabilidad y finanzas; es decir, no se percatan -hasta que ya es demasiado tarde- de la importancia de la gestión. 
También se percibe cierta dificultad para trabajar en equipo, el trabajo colaborativo se vuelve débil; a veces entre los empleados pertenecientes a una misma familia, se da la lucha por el poder y se manifiesta la desconfianza ante el control de la empresa evitando delegar. La contraparte, defiende la cultura del clan, donde las personas comparten mucho de sí mismas (Salas, García y Murillo, 2017). A pesar de las dificultades que enfrentan las MIPYME, sus empresarios, deben implementar pautas internas que mejoren sus diferentes procesos para reflejar positivamente su esfuerzo en los resultados de ventas, el nivel de satisfacción de los clientes y de sus mismos empleados (Vega, Martínez y Parga, 2019). Si bien es cierto que las estructuras organizacionales son marcadas por el tamaño y abren una brecha que identifica la eficiencia de la gestión, nada es imposible para que una microempresa desde su tribuna pueda enfocarse a las áreas que definen su competitividad.

Las MIPYME deben enfocarse a la visión de alcanzar un nivel competitivo, aprovechar su fortaleza de poseer el control directo sobre las operaciones de su negocio. De esta manera lograrán afianzarse en el mercado. Además, si promueven e impulsan la inversión en el aprendizaje, estarán abiertos a nuevas ideas para generar cambios (Vega et al., 2019). Una forma de entender y prestar atención a todas las áreas es la capacitación en la gestión empresarial. Está demostrado que si las organizaciones desarrollan sus capacidades dinámicas (de adaptación al entorno, innovación y capacidad de absorción) podrán responder a los cambios que se susciten en el entorno en que se desenvuelven (Barrios, Olivero y Figueroa, 2020; Feijoo y González, 2020).

La exploración del mercado en que compiten, la observación del proceder de la competencia, el interés por conocer los indicadores económicos, aspectos sociales, políticos y las novedades en tecnología, se reflejarán en la creación de valor en sus actividades, productos, procesos y servicios. Acciones que seguramente redundarán en más ventas, más clientes, más utilidades, mejor desempeño (Olea, Contreras y Barceló, 2016). En este sentido, la idea es explorar el entorno de la pandemia que se vive, no solo para detectar los daños ocasionados, sino también para detectar oportunidades y generar nuevas formas de hacer negocios. Por ejemplo, los empresarios cambiaron la forma de comercializar y distribuir sus productos. Encontraron en las redes sociales y plataformas de Internet, un medio para la continuidad de su negocio.

\subsection{Uso de redes sociales (RS)}

Las empresas enfrentan desafíos en un contexto altamente competitivo donde el uso de la tecnología es un medio que puede significar un aumento en la modernización y agilización de su operación, lo que repercutirá en un aumento en la competitividad (Aguilera, Ávila y Solano, 2017; Real, Leyva y Heredia, 2018). Castellanos (2012) asegura que la inmediatez de la información a través de aplicaciones tecnológicas da a las empresas la oportunidad de mejorar el servicio y aumentar la calidad; además diferentes aplicaciones disponibles en la red permiten acercamiento con sus clientes y proveedores. El uso de la tecnología desencadena al

Revista de Comunicación de la SEECI. 2021, nº 54, 97-118 
interior de la empresa un efecto de cambio y mejora, que la fortalece y contribuye a su éxito, buen desempeño y supervivencia (Mojica y Martínez, 2017).

Las RS como parte de las nuevas tecnologías han asumido un papel determinante que está cambiando la forma de implementar estrategias comerciales. Además de ser un medio que facilita la comunicación y la difusión de información, son una importante alternativa para la implementación de estrategias de marketing (Fonseca, 2014; Palacios, Soto y Merigo, 2015; Pico y Coello, 2017; Villagómez y Acosta, 2020) ya que permiten la interacción masiva y directa con los clientes para difundir sus servicios y productos (Akdogan y Altuntas, 2015).

En las empresas, el uso de RS puede mejorar el desempeño, describir y promover productos o servicios y desarrollar ideas innovadoras de negocios (Tussyadiah y Zach 2013). En los consumidores, este medio ayuda a investigar características propias del producto o servicio, encontrar productos y servicios requeridos; incluso en algunas ocasiones, gracias a las RS los mismos consumidores son un papel activo en la promoción de innovaciones (Pico y Coello, 2017; Sigala y Chalkiti, 2012).

El uso de las RS favorece la detección de ideas propicias para la innovación con un mínimo costo y esfuerzo (Audretsch, Kuratko y Link, 2016). Por medio de las RS, las empresas exploran el entorno en que participan y extraen conocimiento nuevo que mejora las habilidades de los empleados para crear valor en los productos y servicios, atender mercados diversos y complejos (Camisón y Forés, 2010) que implican satisfacer las necesidades de los consumidores cada vez más exigentes (Díaz, et al., 2020; Palacios, et al., 2020). Las RS forman parte de la estructura de una red virtual que apoya el proceso de innovación a largo plazo y puede hacerlo lo suficientemente exitoso (Scuotto, Ferraris y Bresciani, 2016).

El uso de las RS ofrece grandes oportunidades a las pequeñas empresas, permitiéndoles comunicar su mensaje globalmente a bajo costo a través de herramientas Web 2.0 como sitios web y redes sociales (Rivera, Gutiérrez y Olvera, 2020). Scuotto et al. (2017) defienden que las RS mejoran la gestión del conocimiento interno y externo, su adecuado uso reduce la incertidumbre en las inversiones en innovación. Por su parte, Zhou, Wu y Luo (2007) defienden la postura de las RS como mecanismo de las PYME (Pequeñas y medianas empresas) para mejorar el proceso de internacionalización. Las PYME acuden al uso de RS con fines de mercadotecnia, captar nuevos clientes, interactuar con sus clientes y proveedores, así como para incrementar su audiencia (Ainin et al., 2015) en la búsqueda de innovaciones en sus productos o servicios (Scuotto et al., 2017).

El efecto del uso de las RS en las PYME ha sido estudiado a partir de la cantidad de ventas logradas, la rentabilidad generada, la participación de mercado, el posicionamiento obtenido, la satisfacción de los clientes, la eficiencia en los procesos, la calidad del producto, la imagen de la empresa, la adecuada organización de tareas, la rapidez de respuesta, la motivación de los trabajadores, entre otros (Aldape, Abrego y Medina, 2016; Demuner, 2011). Las redes sociales preferidas por las MIPYME para actividades de marketing son: Facebook (81.3\%), seguido 
por Whatsapp (79.8\%); el tercer lugar lo ocupó Youtube (52.80\%); cuarto lugar Facebook Messenger (45.7\%) (iLifebelt, 2017).

\section{OBJETIVO}

Aunque la digitalización muestra un avance importante en su cobertura en los últimos años, para las PYME, la brecha tecnológica aún es alta al compararse con empresas grandes. La penetración de la PYME a la digitalización aún es deficiente, existen grandes rezagos, incluso desde la incorporación de tecnologías digitales maduras, como la disponibilidad de sitios Web, uso del correo electrónico y plataformas digitales (Heredia, 2020; OCDE, 1919). En 2020, debido a la pandemia provocada por COVID-19, la digitalización fue una opción para contrarrestarla. El uso de las RS y plataformas digitales se incrementó (Katz, Jung y Callorda, 2020).

En este contexto, el objetivo de la presente investigación es analizar el uso de las redes sociales que eligieron las microempresas en respuesta a los efectos causados por la pandemia COVID-19 en una localidad mexicana. Se pretende aportar evidencia empírica que ayude al desempeño de la microempresa, que ha sido limitado durante la contingencia sanitaria.

\section{METODOLOGÍA}

Para cumplir el objetivo de esta investigación, se acudió a un estudio transversal con información recabada entre marzo y mayo 2020. Se diseño un cuestionario para contestar en línea. Se envió una invitación para contestar la encuesta a los "contactos" de dos grupos de WhatsApp. Estos grupos son liderados por un empresario. El objetivo de los grupos ha sido apoyarse en la promoción, comercialización y distribución de sus productos, entre otras actividades, como respuesta a las afectaciones provocadas por la pandemia COVID-19. El cuestionario también fue enviado a empresarios de micronegocios registrados en el directorio de la Cámara de Comercio de Córdoba.

El diseño del cuestionario se basó, en primer lugar, en la información recabada de tres entrevistas semi estructuradas a tres empresarios locales. En segundo lugar, se usó esa información y se complementó con los trabajos de Valdez et al. (2012), Díaz (2014), CEPAL (2020a) y Strauss (2012). Finalmente, el cuestionario integró 14 preguntas cerradas de opción múltiple. La primera parte del cuestionario integró dos preguntas para recabar datos sociodemográficos de los participantes. La segunda parte, integró cuatro preguntas encaminadas a identificar los efectos causados por el COVID-19, una pregunta sobre la afectación a los ingresos, dos preguntas encaminadas a detectar actividades de innovación y cinco preguntas referentes al uso de las RS.

Para el análisis de los resultados, se acudió específicamente a un estudio descriptivo que permitió relatar el efecto causado por la pandemia en la actividad empresarial y el uso que los microempresarios dieron a las RS y plataformas 
digitales. La siguiente sección explica los resultados y su contraste con otros estudios. Su presentación sigue el orden de las preguntas del cuestionario.

\section{RESULTADOS Y DISCUSIÓN}

La aplicación del cuestionario redundó en 84 respuestas: el $30 \%$ de los encuestados se dedica a la prestación de servicios personales y profesionales, el $32 \%$ se dedica al comercio y el $33 \%$ se dedica a elaborar sus productos. Los productos manufacturados que resaltan son derivados del uso de madera $61 \%$ y los dirigidos a la elaboración de productos alimenticios (32\%) (Gráfico 1 y 2).

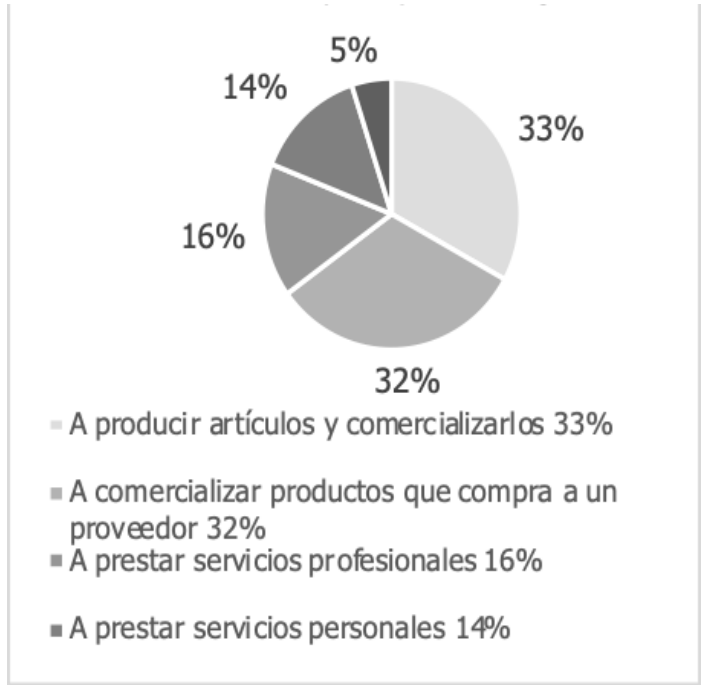

Gráfico 1: Giro principal del negocio Fuente: Elaboración propia

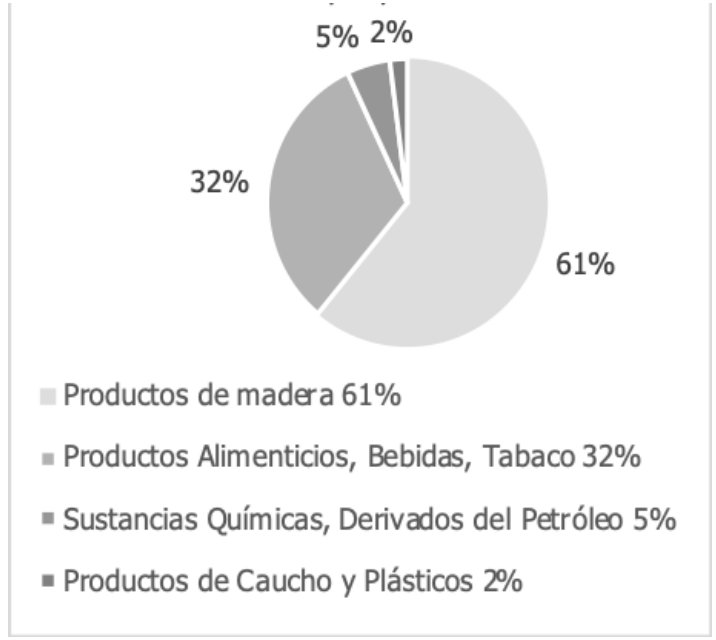

Gráfico 2: ¿A qué rama pertenece el artículo que produce?

Fuente: Elaboración propia

El $50 \%$ de los microempresarios encuestados no cuenta con personal; el resto tuvo que despedir al $16 \%$ de sus empleados por falta de capital de trabajo para pagar sus sueldos (Gráfico 3). Situación similar se presentó en el estudio realizado a PYME mexicanas por $\mathrm{GS}^{2}{ }^{2}$ y $\mathrm{AMVO}^{3}$ (2021), donde el despido permanente de las empresas encuestadas resultó ser $12 \%$ y los trabajadores que conservaron el empleo (31\%) aceptaron la disminución de sus sueldos y prestaciones. La pandemia sobrepasó la importancia del recurso humano como un insumo necesario para acometer cualquier objetivo de la organización (Mojica y Martínez, 2017). Una peculiaridad de las microempresas es el personal compuesto en parte por la familia o por el mismo propietario (Zúñiga et al., 2015), en este caso, se afectó no solo a la empresa, sino también al núcleo familiar.

${ }^{2}$ GS1 México (The Global Language of Business) ha operado más de 30 años como el organismo empresarial que facilita el comercio tradicional y electrónico. Se destaca por asignar el Código de Barras y administrar el sistema de identificación del país.

${ }^{3}$ AMVO (Asociación Mexicana de Venta Online) es una organización civil sin fines de lucro constituida en 2014 con el propósito de apoyar e impulsar el desarrollo del Comercio Electrónico en México.

Revista de Comunicación de la SEECI. 2021, nº 54, 97-118 
En Córdoba, como en otras partes del mundo, la contingencia obligó a los empresarios a replantear o suspender su forma de hacer negocios. El 24 de marzo México entró en la fase 2 de la pandemia y el gobierno estableció la "Jornada Nacional de Sana Distancia" para promover medidas básicas de higiene y distanciamiento social, urgiendo a los ciudadanos a quedarse en casa (\#quédateencasa) y suspender actividades no esenciales en los sectores público, privado y social (Clavellina, 2020). La pandemia obligó al $48 \%$ de las empresas de este estudio a cerrar su negocio. $11 \%$ argumentó haber cerrado para seguir los lineamientos del aislamiento social, 7\% obligadas por las autoridades. Y quizá, la causa más grave, fue cerrar el negocio debido a la disminución de ventas y clientes (30\%) (Gráfico 4). Similar al $25 \%$ reportado por la encuesta de GS1 y AMVO (2021), cuyas PYME encuestadas también cerraron sus establecimientos a causa del COVID-19.

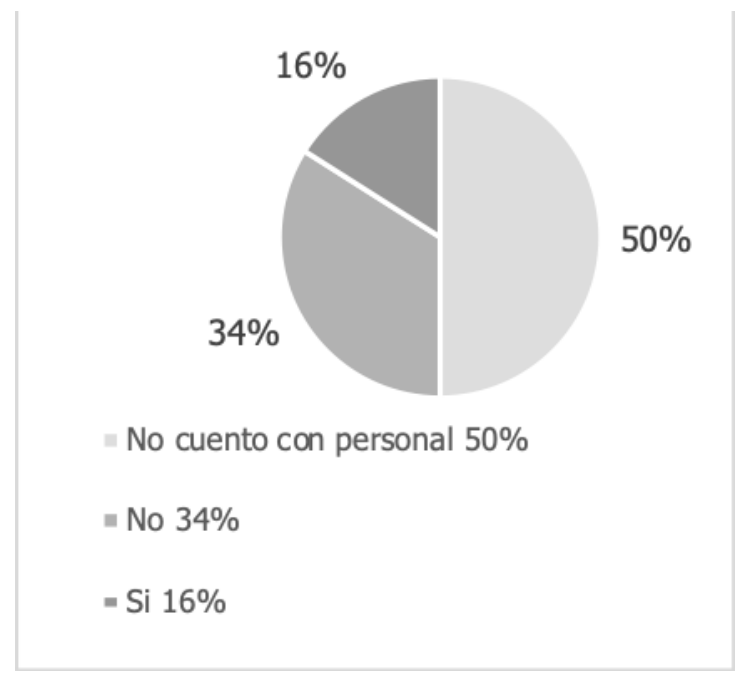

Gráfico 3: ¿Despidió empleados?

Fuente: Elaboración propia

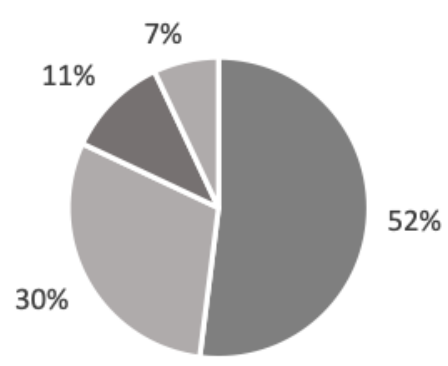

- No cerré mi negocio $52 \%$

- Si debido a la disminución de clientes y ventas 30\%

- Si para tomar precauciones sanitarias $11 \%$

" Si obligado por las autoridades $7 \%$

Gráfico 4: ¿Cerró su negocio?

Fuente: Elaboración propia

Ante la disminución de sus flujos de efectivo y la oferta de apoyo del gobierno, $52 \%$ de los empresarios se apresuraron a ingresar solicitudes para recibir los beneficios, pero solo el $22 \%$ de ellos lo logró. El $30 \%$ de las microempresas solicitantes fueron rechazados debido al ingreso de documentación incorrecta, aunado al inmediato cierre de la plataforma que recibía las solicitudes (Gráfico 5). En condiciones normales, cuando a las MIPYME les ofrecen algún crédito bancario, según ENAPROCE (2019), 7 de cada 10 no lo acepta, la razón principal es el costo de financiamiento y la complejidad de los requisitos solicitados.

En sí, el financiamiento es uno de los principales problemas que enfrentan las MIPYMES en México (Demuner, 2011). Aunque durante la pandemia, independientemente del tamaño, las empresas enfrentaron importantes dificultades para el acceso al crédito (CEPAL, 2020a), las más pequeñas fueron las empresas más vulnerables. $73 \%$ de las PYME reportó impacto en su flujo de efectivo debido a la suspensión de su actividad, por el retraso en el pago de sus clientes, cancelaciones de pedidos, entre otros (GS1 y AMVO, 2021). Para ello, aunque el gobierno y el 
sistema financiero instrumentaron algunas políticas de apoyo entre las que resaltan los micro préstamos (Clavellina, 2020), cuya aceptación prometía ser rápida, pocas empresas fueron beneficiadas.

Debido a la complejidad de los requisitos, es común que las MIPYME busquen financiamiento entre sus amigos y/o familiares. Alternativa que coincide con sus intereses particulares, en el sentido que los dirigentes de estas empresas tienden a gestionar mínimamente el riesgo (Pereira y Cuero, 2018). De los empresarios encuestados el $45 \%$ solicitó préstamos a familiares y/o amigos (20\% si lo obtuvo y $25 \%$, no) (Gráfico 6). En el caso de GS1 y AMVO (2021), el $43 \%$ de sus encuestados solicitaron préstamos.

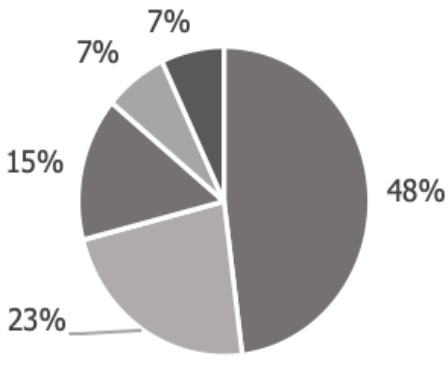

- No solicite préstamo a externos $48 \%$

- Si, pedí apoyo del gobierno y NO me lo dieron $23 \%$

- Si, al banco y SI me lo prestaron $15 \%$

- $\mathrm{Si}$, al banco y No me lo dieron $7 \%$

- Si, pedí apoyo del gobierno y SI me lo dieron 7\%

Gráfico 5: ¿Solicitó algún préstamo a externo?

Fuente: Elaboración propia

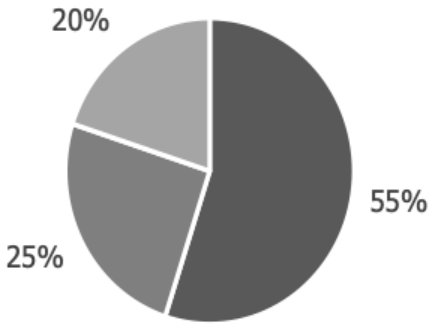

- No solicité préstamo a familiares y/o amigos $55 \%$

- $\mathrm{Si}$, pero NO me lo prestaron $25 \%$

= Si, y SI me lo dieron $20 \%$

Gráfico 6: ¿Solicitó algún préstamo a familiares?

Fuente: Elaboración propia

Las predicciones de la OECD (2020) respecto a la gravedad en la economía ocasionada por COVID-19 se cumplieron. Al paso de la pandemia, $90 \%$ de los microempresarios encuestados resintieron la disminución en sus ingresos; de ellos, el $66 \%$ sufrió un decremento de más del 31\% (Gráfico 7). La baja demanda, el freno a la producción, la disminución en el suministro de materia prima, la cancelación o retraso de actividades de promoción y problemas de logística y entrega, fueron las principales causas argumentadas por los empresarios.

Solo el $16 \%$ de los microempresarios buscaron como alternativa para obtener ingresos, incursionar en otro tipo de producto; de este $16 \%$, solo $2 \%$ intentó la innovación incremental (Gráfico 8). Estos resultados son críticos, los empresarios no reaccionaron con innovación ante los cambios del medio ambiente (Rodríguez y Da Cunha, 2018). En línea con la prospectiva del emprendimiento intensivo en conocimiento, las empresas tienen un espíritu emprendedor dinámico que lidera su capacidad para explotar oportunidades usando conocimiento externo intensivo en combinación con el conocimiento interno para introducir innovación (Audretsch et al., 2016). En este contexto la adquisición del conocimiento, definida como la habilidad de la empresa para capturar y apropiarse de información del exterior (Feijoo y 
González, 2020) permite al empresario captar ideas novedosas, explorar la competencia, conocer indicadores macroeconómicos, conocer los avances tecnológicos, etc. Sin embargo, en este estudio, quizá por la precipitación en el cambio de estrategia para recuperar ventas, el $84 \%$ no incursionó en innovación (Gráfico 8 ) y del bajo porcentaje que sí lo hizo (2\%), el $84 \%$ no tuvo éxito con su producto innovador (Gráfico 9).

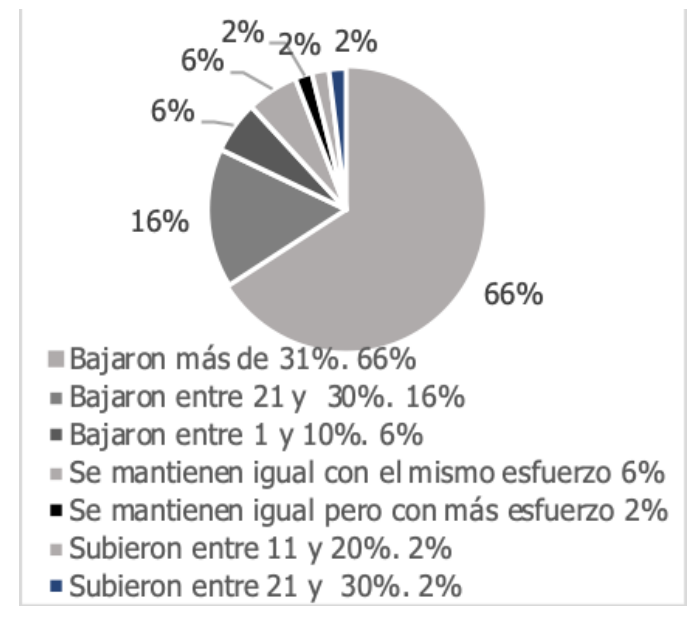

Gráfico 7: ¿Qué ocurrió con sus ingresos? Fuente: Elaboración propia

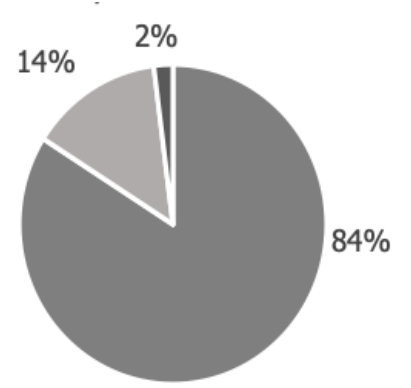

- No $84 \%$

- Si por un producto que ya existia en el mercado $14 \%$

- Si por un producto o servicio que ya existia en el mercado, pero yo lo mejoré $2 \%$

\section{Gráfico 8: ¿Cambió su producto o servicio por otro?}

Fuente: Elaboración propia

A raíz de la pandemia, 3 de cada 10 empresarios iniciaron o incrementaron el uso de las RS (Gráfico 10). En primer lugar, fueron usadas para lograr una presencia digital que les permitiera compartir información de valor acerca de su empresa y su producto, publicitar su producto o servicio, atraer clientes y desencadenar posibles compras, además usaron la información del perfil de los usuarios y sus relaciones; de esta forma sí un anuncio llegara a un miembro de una red que lo considera interesante, lo compartiría con sus contactos, provocando una distribución viral. El $70 \%$ de los encuestados, antes de la contingencia ya usaban redes sociales. Dichas empresas saben que tener una presencia digital es una obligatoriedad en la actualidad.

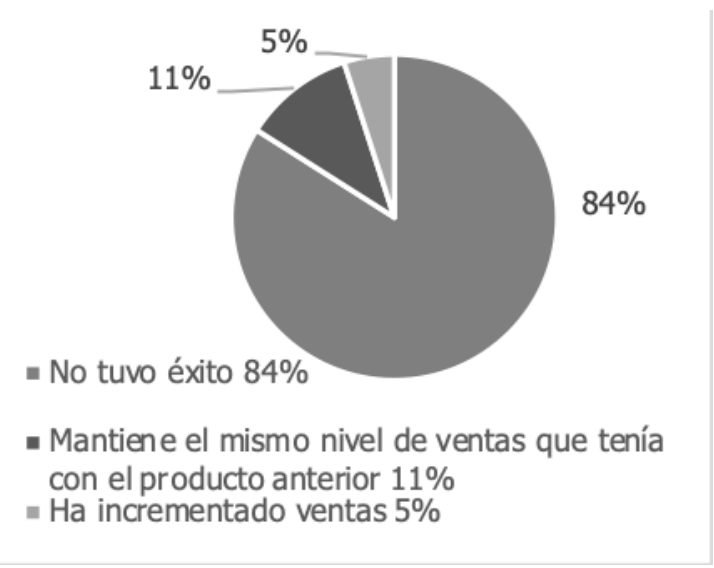

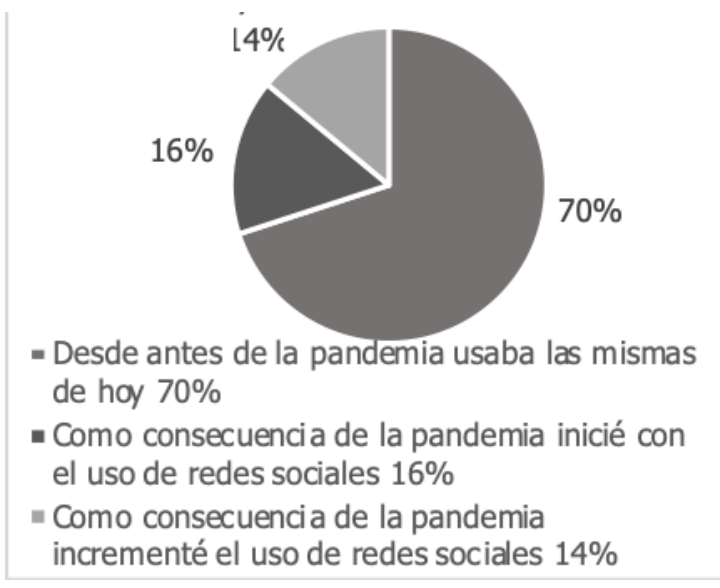


Gráfico 9: Ese producto que cambio ¿Qué efecto le generó al comercializarlo?

Fuente: Elaboración propia
Gráfico 10: ¿Desde cuándo usa redes sociales para promover sus productos?

Fuente: Elaboración propia

Los empresarios encuestados reportaron los beneficios que obtuvieron del uso de las RS, entre ellos: seguimiento a sus clientes (24\%), incremento de clientes (19\%), posicionar productos $\mathrm{y} / \mathrm{o}$ servicios (15\%) y acceder a nuevos mercados (15\%) (Gráfico, 11). Resultados coincidentes con el estudio de PYME de González y Flores (2020) que también reaccionaron a la contingencia COVID-19 empleando una serie de estrategias empresariales alineadas al uso del comercio electrónico y uso masivo de las redes sociales. Una vez más se confirma que las RS son importantes herramientas de fidelización, que generan enlaces externos que permiten obtener retroalimentación de los productos y servicios (Díaz, 2014). Correa (2020) sugiere su utilización para el incremento en ventas y un mejor manejo de la identidad empresarial.

Si bien las RS atraen a los empresarios para atender y mantener a sus clientes satisfechos, así como conseguir fidelizarlos para conducir al negocio a ser sostenible, exitoso y consciente (González y Flores, 2020; Real et al., 2018), no pueden evitar reconocer que deben invertir tiempo en su uso (32\%), se distraen en sus actividades (31\%) y temen divulgar información de su negocio (12\%) (Gráfico 12). Al respecto, Strauss (2012) sugiere a los propietarios de las pequeñas empresas, usar la norma Pareto 80-20: el 80\% del contenido de la información que se distribuye a través de las redes sociales debe ser acerca de los clientes, porque lo que importa es conocer sus intereses, cuáles son sus necesidades y nuevas soluciones para sus problemas; lo importante es investigar qué se puede vender a alguien que se desconoce lo que está dispuesto a comprar; y el $20 \%$ solo debe ser información del negocio (Strauss, 2012).

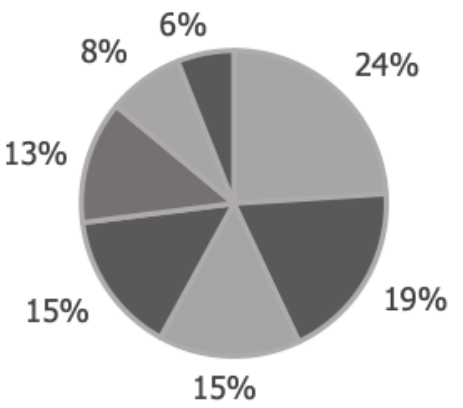

Dar seguimiento a los clientes $24 \%$ - Incrementar los clientes $19 \%$

Posicionar mi producto/servicio $15 \%$ - Uegar a nuevos mercados $15 \%$ a Posicionar mi empresa $13 \%$ - Mayores ventas $8 \%$

Gráfico 11: Beneficios en el uso de redes sociales

Fuente: Elaboración propia

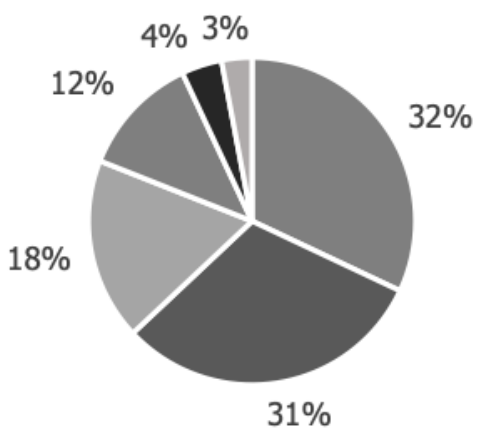

- Inversión de tiempo 32\%

- Distracción 31\%

- Ninguna $18 \%$

- Divulgación de información del negocio 12\%

- Incremento en gastos $4 \%$

- Improductividad 3\%

Gráfico 12: Desventajas en el uso de redes sociales

Fuente: Elaboración propia 
El uso de las redes sociales es popular. Según el reporte anual de The Global State of Digital, en 2020 el 52\% de la población mundial utiliza las redes sociales, para la comunicación entre personas y para posicionar una empresa -sin importar su tamaño- (Digital, 2020). México cuenta con una población de 128.3 millones de habitantes, 114.3 millones usan conexión móvil, 89.00 millones son usuarios de Internet y 89 millones usan redes sociales (69\%) (Digital, 2020a). De las redes sociales más usadas en el mundo, Facebook, YouTube y WhatsApp, ocupan los tres primeros lugares, situación similar en México (Tabla 2) y en el presente estudio.

Tabla 2. Redes sociales más usadas.

\begin{tabular}{ll}
\hline \multicolumn{1}{c}{ En el mundo } & \multicolumn{1}{c}{ En México } \\
\hline 1. Facebook: 2.449 millones & 1. YouTube $96 \%$ \\
2. YouTube: 2.000 millones & 2. Facebook $94 \%$ \\
3. WhatsApp: 1.600 millones & 3. WhatsApp $89 \%$ \\
4. FB Messenger: 1.300 millones & 4. FB Messenger $78 \%$ \\
5. WeChat: 1.151 millones & 5. Instagram $71 \%$ \\
6. Instagram: 1.000 millones & 6. Twitter $61 \%$ \\
7. TikTok: 800 millones & 7. Pinterest $46 \%$ \\
8. QQ: 731 millones & 8. Linkedin $36 \%$ \\
9. Ozone: 517 millones & 9. Snapchat $35 \%$ \\
10. Sina Weibo: 497 millones & 10. Skype $32 \%$ \\
11. Reddit: 430 millones & 11. Tumblr $22 \%$ \\
12. Snapchat: 382 millones & 12. Twich $22 \%$ \\
13. Twitter: 340 millones & 13. WeChat $21 \%$ \\
14. Pinterest: 322 millones & 14. TikTok $20 \%$ \\
15. Kuaishou: 316 millones & 15. Reddit $17 \%$ \\
& 16. Line $16 \%$ \\
\hline
\end{tabular}

Fuente: Elaboración propia de Digital (2020, 2020a). Diapositivas 11/92 y 43/92.

En este estudio, las MIPYME usan las redes sociales como primer paso para vender sin intermediarios, asimismo acuden a plataformas digitales, por ejemplo, Mercado libre y Amazon, por ser las más populares. El grupo encuestado, acudió en primer lugar a Facebook (32\%), resultado coincidente con los obtenidos por Aldape et al. (2016) el uso de Facebook es una herramienta que fomenta las actividades de marketing. En segundo lugar, usaron WhatsApp (30\%), seguido de Instagram (13\%). Los resultados obtenidos confirman los beneficios del uso de las RS: acercamiento a consumidores, promoción y difusión de productos y servicios (Díaz et al., 2020; Villagómez y Acosta, 2020) con bajos costos (Correa, 2020) (Gráfico 13).

Los empresarios reconocen que WhatsApp les ha aportado mayores beneficios (36\%), esto se explica a la ayuda que recibieron los empresarios encuestados al ser invitados por un líder a conformar grupos para promover sus productos y usar esta red como comunicación directa con sus clientes. Facebook (34\%) y correo electrónico (24\%), también participaron como herramientas tecnológicas en la ayuda a estos empresarios durante la pandemia (Gráfico 13). 


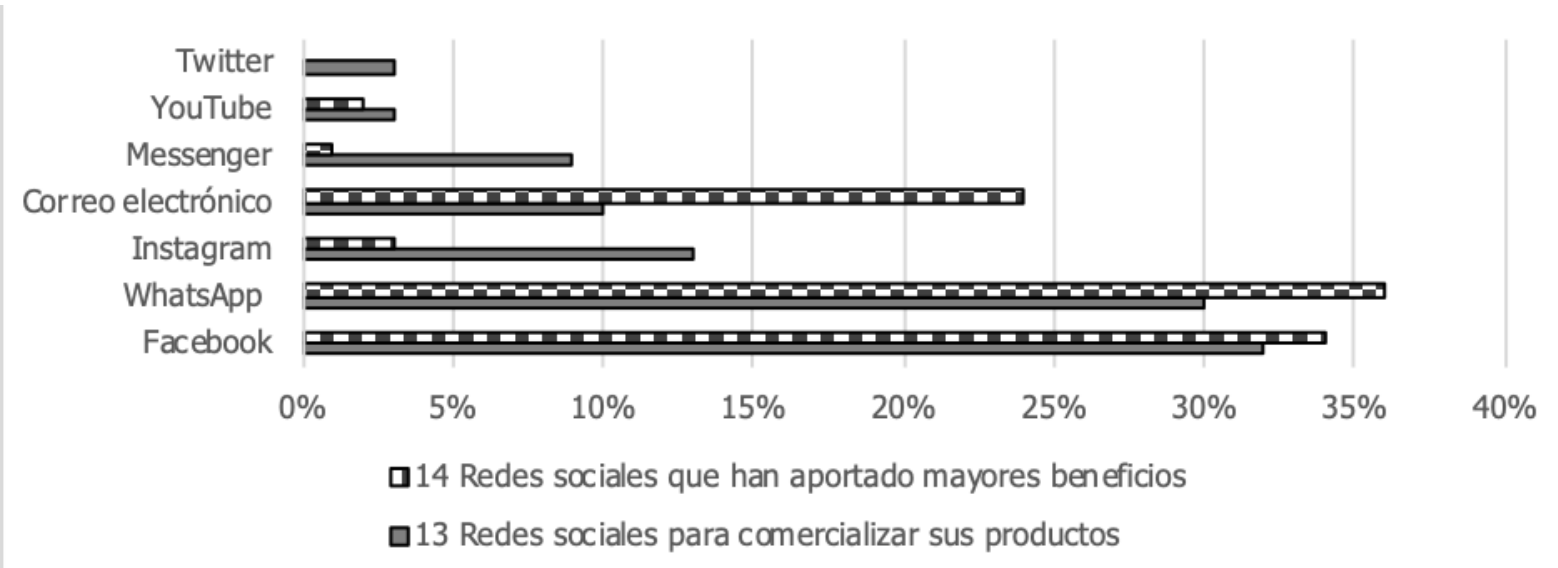

Gráfico 13: Redes sociales

Fuente: Elaboración propia

\section{CONCLUSIONES}

La magnitud de los efectos provocados por la pandemia COVID-19, se considera la mayor crisis económica en el mundo desde los años treinta. Todas las economías han sufrido las consecuencias, unos países más que otros, unas empresas más que otras, pero especialmente afectados han sido los grupos vulnerables. Aunque el gobierno y organismos mundiales han implementado diferentes estrategias para disminuir las pérdidas humanas y económicas, el futuro aún genera incertidumbre.

Si bien es cierto que la contingencia ha afectado al sector empresarial de manera relevante, las microempresas han sufrido pérdidas importantes. Es obvia la reflexión que en este sector participan familias completas de pocos recursos que dependen únicamente de los ingresos generados por su negocio. A pesar de las medidas implementadas por el gobierno mexicano en apoyo a la MIPYME, aún hay camino por andar.

El presente estudio, bajo el auspicio de una metodología deductiva describió los resultados encontrados, aporta empíricamente efectos negativos que se acumulan a las debilidades de las MIPYME. La contingencia afectó aun más su desempeño, mermando sus ingresos en más de un $31 \%$. En la búsqueda de medidas generadoras de ingresos, solo el $16 \%$ de estos empresarios incursionaron en otro producto; el uso de las redes sociales se incrementó en 30\% como un aliciente para la promoción de productos y servicios, incrementar y dar seguimiento a sus clientes, posicionar su producto e incursionar en otros mercados. Las redes sociales preferidas por este grupo no distan de la generalidad, Facebook, Instagram y WhatsApp, siendo esta última la red que aportó mayores beneficios.

Además, con las implicaciones prácticas de este estudio, se da a conocer la importancia y relevancia del uso de las redes sociales que han manifestado los micronegocios en Córdoba. Este estudio contribuye a la investigación empírica de las 
MIPYME, aporta evidencia para potencializar el uso de nuevas tecnologías para minimizar sus debilidades y sostener o incrementar sus fortalezas.

Se reconoce como limitación del estudio el uso de una muestra relativamente pequeña y dirigida sólo a microempresarios de una localidad. Se visualiza la necesidad de realizar investigaciones con MIPYME sobre posibles relaciones del uso de las redes sociales con variables como capacitación, cultura, gestión de conocimiento, procesos de innovación, etc. que participen en ideas para fomentar su sustentabilidad económica.

\section{REFERENCIAS}

Ainin, S., Parveen, F., Moghavvemi, S., Jaafar, N. I. \& Shuib, N. L. M. (2015). Factors influencing the use of social media by SMEs and its performance outcomes. Industrial Management and Data Systems, 115(3), 570-588. https://doi.org/10.1108/IMDS-07-2014-0205

Aguilera, A., Ávila, P. \& Solano, J. (2017). Las TIC en la formulación estratégica de las pymes de Santiago de Cali - Colombia. Entramado, 13(1), 102-111.

Akdogan, M. \& Altuntas, B. (2015). Covert Marketing Strategy and Techniques. Procedia-Social and Behavioral Sciences, 207, 135-148. https://doi.org/10.1016/j.sbspro.2015.10.162

ALAMPYME. (2020). Asociación latinoamericana de micros, pequeños y medianos empresarios. https://integraa.org/2020/03/asociacion-latinoamericana-de-microspequenos-y-medianos-empresarios-alampyme Consultado el 20 de mayo de 2020.

Aldape, N. K. A., Abrego, A. D. \& Medina, Q. J. M. (2016). Análisis de la percepción de uso de las redes sociales como herramienta de marketing en las MiPYMES de Tamaulipas. RISTI, No. 18(6), 49-65. http://dx.doi.org/10.17013/risti.18.49-65.

AMIA. (2020). Asociación Mexicana de la Industria Automotriz. Noticias. http://www.amia.com.mx/. Consultado el 20 de mayo de 2020.

Audretsch, D. B., Kuratko, D. F. \& Link, A. N. (2016). Dynamic entrepreneurship and technology-based innovation. Journal of Evolutionary Economics, 26, 603-620. https://doi.org/10.1007/s00191-016-0458-4

Barrios, H. K. C., Olivero, V. E. \& Figueroa, S. B. (2020). Condiciones de la gestión del talento humano que favorecen el desarrollo de capacidades dinámicas. Información tecnológica, 31(2), 56-62. http://dx.doi.org/10.4067/S0718$\underline{07642020000200055}$

Camisón, C. \& Forés, B. (2010). Knowledge absorptive capacity: New insights for its conceptualization and measurement. Journal of Business Research, 63, 707-715.

Revista de Comunicación de la SEECI. 2021, nº 54, 97-118 
Demuner Flores, M. R. Uso de redes sociales en microempresas ante efectos COVID-19

Castellanos, P. (2012). La nueva forma de servicio al cliente. Revista Merca 2.0. 10(119), 60-61.

CEPAL. (Comisión Económica para América Latina y el Caribe). (2020). América Latina y el Caribe ante la pandemia del COVID-19. Efectos económicos y sociales. Informe especial COVIT-19, No. 1. Editorial: CEPAL, 3 de abril. https://www.cepal.org/es/publicaciones/45337-america-latina-caribe-la-pandemiacovid-19-efectos-economicos-sociales

CEPAL. (Comisión Económica para América Latina y el Caribe). (2020a). Dimensionar los efectos del COVID-19 para pensar en la reactivación. Informe especial COVIT19. No. 2. Editorial: CEPAL, 20 de abril. https://www.cepal.org/es/publicaciones/45445-dimensionar-efectos-covid-19pensar-la-reactivacion

Clavellina, M. J. L. (2020). Medidas de contención y de política económica ante la pandemia de COVID-19 en México. Instituto Belisario Domínguez, Senado de la República, Dirección General de Finanzas, No. 94, 1-9. http://bibliodigitalibd.senado.gob.mx/handle/123456789/4867

Correa, G. L. A. (2020). Relación entre la tecnología y la comercialización en la PyME ubicada en Zacatecas, Mercados y Negocios, 1(41), enero-junio, 107-124. https://dialnet.unirioja.es/servlet/articulo?codigo $=7430148$

Demuner, F. M. R. (2011). PYMES Competitivas. Eae Editorial Academia Española.

Demuner, F. M. R., Becerril, T. O. U. \& Ibarra, C. M. A. (2018). Capacidad de respuesta y capacidad de absorción. Estudio de empresas manufactureras en México. Nóesis Revista de Ciencias Sociales y Humanidades, 27(53), 61-77. http://dx.doi.org/10.20983/noesis.2018.4.4.

Díaz, B. J. L., Armas, T. V., Morales, C. J. C. \& Ortigoza, H. A. (2020). Estudio de la mezcla de promoción en las PYMES Poblanas. Revista Relayn Micro y pequeña empresa en Latinoamérica, 4(2), 102112. https://doi.org/10.46990/relayn.2020.4.2.106

Díaz, L. J. A. (2014). Propuesta de red social como herramienta del marketing relacional: fidelización de clientes en pequeñas empresas de calzado. Ciencia $y$ Tecnología, 10(1), 31-46.

Digital. (2020). Global Digital Overview v01. https://www.slideshare.net/DataReportal/digital-2020-global-digital-overviewjanuary-2020-v01-226017535

Digital. (2020a). Digital 2020: México. https://datareportal.com/reports/digital-2020mexico 
Demuner Flores, M. R. Uso de redes sociales en microempresas ante efectos COVID-19

ENAPROCE. (2019). Resultados de la Encuesta Nacional sobre Productividad y Competitividad de las Micro, Pequeñas y Medianas Empresas. INEGI. https://www.inegi.org.mx/programas/enaproce/2018/

Feijoo, P. D. y González, I. M. (2020). Las capacidades dinámicas: análisis de las empresas exportadoras en América Latina. INNOVAR Research Journal, 5(1), 198209. http://dx.doi.org/10.33890/innova.v5.n1.2020.1179

FMI. (Fondo Monetario Internacional) (2020). Fiscal Monitor, abril de 2020. https://www.imf.org/es/Publications/FM/Issues/20 20/04/06/fiscal-monitor-april$\underline{2020}$

Fonseca, A. (2014). Marketing Digital en redes sociales: Lo imprescindible en Marketing Online, Calendar, Palma Área, España.

González, D. R. R. \& Flores, L. K. N. (2020). Cultura organizacional y Sustentabilidad empresarial en las Pymes durante crisis periodos de confinamiento social. Revista Internacional Multidisciplinaria, CIID Journal, No. 1, 28-41.

GS1 México (The Global Language of Business) y AMVO (Asociación Mexicana de Ventas Online). (2020). Estudio sobre venta Online en PyMEs. Segunda edición. www.gs1mexico.org y www.amvo.org.mx/estudios

Heredia, A. (2020). Políticas de fomento para la incorporación de las tecnologías digitales en las micro, pequeñas y medianas empresas de América Latina: revisión de experiencias y oportunidades. Documentos de Proyectos (LC/TS.2019/96), Santiago, Comisión Económica para América Latina y el Caribe (CEPAL).

Ibarra, C. M. A., González, T. L. A. \& Demuner, F. M. R. (2017). Competitividad empresarial de las pequeñas y medianas empresas manufactureras de Baja California. Estudios Fronterizos, 18(35) enero-abril, 107-130. https://doi.org/10.21670/ref.2017.35.a06

iLifebelt. (2017). 70 Estudio de uso de redes sociales en Centro América y el caribe. Engagement República. https://nestoraltuve.files.wordpress.com/2018/02/estudiorsca18.pdf

INEGI. (2014). Censos económicos 2014. Veracruz de Ignacio de la Llave. SNIEG. Información de Interés Nacional. Instituto Nacional de Estadística y Geografía. https://www.inegi.org.mx/contenido/productos/prod serv/contenidos/espanol/bvi negi/productos/nueva estruc/702825083458.pdf

INEGI. (2019). Censos Económicos 2019. Micro, pequeña, mediana y gran empresa : estratificación de los establecimientos. Instituto Nacional de Estadística y Geografía. México: INEGI, c2020. https://www.inegi.org.mx/programas/ce/2019/ 
Demuner Flores, M. R. Uso de redes sociales en microempresas ante efectos COVID-19

INEGI. (2020). Atribuyéndose a los efectos del COVIT-19. Instituto Nacional de Estadística y Geografía. https://www.inegi.org.mx/default.html. Consultado el 11 de mayo.

INEGI. (2020a). Indicador Mensual de la Actividad Industrial, Sistema de Cuentas Nacionales de México. Instituto Nacional de Estadística y Geografía. www.inegi.org.mx/temas/imai/. Consultado el 11 de junio de 2020.

Katz, R., Jung, J. \& Callorda, F. (2020). El estado de la digitalización de América Latina frente a la pandemia del COVID-19. Ed: CAF (Banco de Desarrollo de América Latina), mayo.

Konfio. (2020a). Reporte Pyme 2020: Haciendo frente y pronosticando el futuro. Junio. www.konfio.MX

Mojica, C. E. P. \& Martínez, S. M. C. (2017). Orientación al mercado, innovación y capacidades competitivas, determinantes del desempeño de las PYMEs del Estado de Aguascalientes. RICEA, Revista Iberoamericana de Contaduría, Economia y Administración, 6(11), Enero - Junio. https://doi.org/10.23913/ricea.v6i11.93

OCDE. (Organización para la Cooperación y el Desarrollo Económicos). (2019). América Latina y el Caribe 2019. Políticas para PYMEs competitivas en la Alianza del Pacífico y países participantes de América del Sur. OECD Publishing, París. https://www.oecd.org/publications/america-latina-y-el-caribe-2019-60745031es.htm

OECD. (Organisation for Economic Co-operation and Development). (2020). Interim Economic Assessment, Press conference Laurence Boone, OECD Chief Economist. March, 2th. https://www.oecd.org/economic-outlook/march-2020/ Consultado el 1 de febrero 2020.

OIT (Organización Internacional del Trabajo). (2020). México y la crisis de la COVID19 en el mundo del trabajo: respuestas y desafíos. Feix, N. (Coord.) Panorama laboral en tiempos de COVID-19. Nota técnica. Octubre. https://www.ilo.org/wcmsp5/groups/public/---americas/---ro-lima/---ilomexico/documents/publication/wcms 757364.pdf

Olea, J., Contreras, O. \& Barceló, M. (2016). Las capacidades de absorción del conocimiento como ventajas competitivas para la inserción de pymes en cadenas globales de valor. Estudios Gerenciales, Vol. 32, 127-136. http://dx.doi.org/10.1016/j.estger.2016.04.002

Palacios, M. D., Soto, A. P. \& Merigo, J. M. (2015). Analyzing the effects of technological, organizational and competition factors on Web knowledge exchange in SMEs. Telematics and Informatics, 32(1), 23-32. http://dx.doi.org/10.1016/j.tele.2014.08.003 
Demuner Flores, M. R. Uso de redes sociales en microempresas ante efectos COVID-19

Palacios, M. D., Ponce, A. J., Villamrin , V. W. \& Palma, A. A. M. (2020). Las redes sociales y su influencia como estrategia de marketing en las PYMES de Manabí Ecuador. Revista Observatorio de la Economía Latinoamericana, abril, 1-23. https://www.eumed.net/rev/oel/2020/04/redes-sociales-marketing.pdf

Pereira, O. L. E. \& Cuero, A. Y. A. (2018). Gestión empresarial en la prevención de riesgos por parte de mipymes. SUMA DE NEGOCIOS, 9(19), 68-76. http://dx.doi.org/10.14349/sumneg/2018.V9.N19.A8

Pico, V. L. M. \& Coello, Y. R. R. (2017). Relación entre el ciclo de vida de las Pymes en redes sociales y el emprendimiento en la ciudad de Guayaquil. INNOVA Research Journal, 2(5), 125-136.

Real, P. I, Leyva, C. A. B. \& Heredia, B. J. A. (2018). Uso e impacto de las redes sociales en las estrategias de marketing de las PyME's. Revista De Investigación Académica Sin Frontera, 7(19), Julio-diciembre, 1-24.

Rivera, T. I., Gutiérrez, A. J. \& Olvera, L. M. D. (2020). Websites and Social Networks. A Study of Healthcare SMEs in Andalusia. In: Rocha Á., Ferrás, C., Montenegro, M. C. \& Medina, G. V. (eds) Information Technology and Systems. ICITS 2020. Advances in Intelligent Systems and Computing, vol 1137. Springer, Cham.

Rodríguez, P. L. \& Da Cunha, C. (2018). Impacts of Big Data Analytics and Absorptive Capacity on Sustainable Supply Chain Innovation: a Conceptual Framework. Scientific Journal of Logistics, 14(2), 151-161. https://hal.archivesouvertes.fr/hal-01757102/document

Salas, A. L., García, S. M. \& Murillo, V. G. (2017). Efecto de la cultura organizacional en el rendimiento de las PYMES de Cali. SUMA DE NEGOCIOS, 8, 88-95. http://dx.doi.org/10.1016/j.sumneg.2017.11.006

Scuotto, V., Ferraris, A. \& Bresciani, S. (2016). Internet of things: Applications and challenges in smart cities. A case study of IBM smart city projects. Business Process Management Journal, 22(2), 357-367. 10.1108/BPMJ-05-2015-0074

Scuotto, V., del Giudice, M., della Peruta, M. R. \& Tarba, S. (2017). The performance implications of leveraging internal innovation through social media networks: An empirical verification of the smart fashion industry. Technological Forecasting and Social Change, (120), 184-194. https://doi.org/10.1016/j.techfore.2017.03.021

Sigala, M. \& Chalkiti, K. (2012). Knowledge management and web 2.0: Preliminary findings from the greek tourism industry. In M. Sigala, E. Christou, \& U. Gretzel (Eds.), Web 2.0 in travel, tourism and hospitality: Theory, practice and cases. Farnham: Ashgate Publishers.

Strauss, D. S. (2012). The Small Business Bible. Third Edition. Editorial Wiley

Revista de Comunicación de la SEECI. 2021, nº 54, 97-118 
Demuner Flores, M. R. Uso de redes sociales en microempresas ante efectos COVID-19

Tussyadiah, I. \& Zach, F. (2013). Social media strategy and capacity for consumer co-creation among destination marketing organizations. In L. Cantoni \& Z. Xiang (Eds.), Information and communication technologies in tourism. Berlin: Springer.

Ugalde, N. (2009). El impacto de la cultura en el desarrollo de las PYMES. Ciencias Económicas, 27(1), 293-301. https://revistas.ucr.ac.cr/index.php/economicas/article/view/7131/6815

Valdez, J. L. E., Rascón, R. J. A., Ramos, E. E. A. \& Huerta, G. J. E. (2012). Redes Sociales, una estrategia corporativa para las PyMES de la región de Guaymas Sonora México. Revista FIR, FAEDPYME International Review, 1(1), Enero - Junio, 62-74. http://faedpyme.ojs.upct.es/index.php/revista1/article/view/15

Vega, M. J. E., Martínez, S. M. C. \& Párga, M. N. (2019). Influencia del aprendizaje organizacional y los resultados de las Pymes. Investigación Administrativa, 48(124). http://www.redalyc.org/articulo.oa?id=456059299003.

Villagómez, M. A. R. \& Acosta, G. E. (2020). Uso de redes sociales digitales como estrategia de mercadotecnia en pymes pirotécnicas de Tultepec en el Estado de México. Revista de Comunicación de la SEECI, (52), 73-93. doi: http://doi.org/10.15198/seeci.2020.52.73-93

Zhou, L., Wu, W. P. \& Luo, X. (2007). Internationalization and the performance of born-global SMEs: The mediating role of social networks. Journal of International Business Studies, 38(4), 673-690. https://doi.org/10.1057/palgrave.jibs.8400282

Zúñiga, J. S., Soza, A. S. \& Soria, B. K. (2015). Dinámica del emprendimiento y el desempleo en la Patagonia Chilena. Magallania, 43(1), 103115. http://dx.doi.org/10.4067/S0718-22442015000100007

\section{AUTOR:}

\section{María del Rosario Demuner Flores}

Doctora en Ciencias Económico Administrativas. Docente e investigadora en la Coordinación de Investigación y Estudios Avanzados de la Facultad de Contaduría y Administración. Miembro del Sistema Nacional de Investigadores CONACYT. mrdemunerf@uaemex.mx

demuner7@yahoo.com

Orcid ID: https://orcid.org/0000-0002-4542-9113

ResearchGate ID: https://www.researchgate.net/profile/Maria Demuner-Flores

Autores Redalyc: https://www.redalyc.org/autor.oa?id=320

Google Scholar: https://scholar.google.com/citations?user=FfxkejUAAAAJ\&hl=es 